\title{
Phase-engineered bosonic quantum codes
}

\author{
Linshu Li $\odot,{ }^{1}$ Dylan J. Young, ${ }^{1,2}$ Victor V. Albert,,${ }^{1,3,}{ }^{*}$ Kyungjoo Noh, ${ }^{1,4}$ Chang-Ling Zou, ${ }^{1,5}$ and Liang Jiang ${ }^{1,4,6}$ \\ ${ }^{1}$ Yale Quantum Institute, Department of Applied Physics and Department of Physics, Yale University, New Haven, Connecticut 06511, USA \\ ${ }^{2}$ JILA, NIST, and Department of Physics, University of Colorado, 440 UCB, Boulder, Colorado 80309, USA \\ ${ }^{3}$ Institute for Quantum Information and Matter and Walter Burke Institute for Theoretical Physics, \\ California Institute of Technology, Pasadena, California 91125, USA \\ ${ }^{4}$ AWS Center for Quantum Computing, Pasadena, California 91125, USA \\ ${ }^{5}$ Key Laboratory of Quantum Information, University of Science and Technology of China, Hefei, Anhui 230026, China \\ ${ }^{6}$ Pritzker School of Molecular Engineering, University of Chicago, 5640 South Ellis Avenue, Chicago, Illinois 60637, USA
}

(Received 1 January 2021; accepted 2 June 2021; published 29 June 2021)

\begin{abstract}
Continuous-variable systems protected by bosonic quantum codes have emerged as a promising platform for quantum information. To date, the design of code words has centered on optimizing the state occupation in the relevant basis to generate the distance needed for error correction. Here, we show tuning the phase degree of freedom in the design of code words can affect, and potentially enhance, the protection against Markovian errors that involve excitation exchange with the environment. As illustrations, we first consider phase engineering bosonic codes with uniform spacing in the Fock basis that correct excitation loss with a Kerr unitary and show that these modified codes feature destructive interference between error code words and, with an adapted "twolevel" recovery, the error protection is significantly enhanced. We then study protection against energy decay with the presence of mode nonlinearities and analyze the role of phase for optimal code designs. We extend the principle of phase engineering to bosonic codes defined in other bases and multiqubit codes, demonstrating its broad applicability in quantum error correction.
\end{abstract}

DOI: 10.1103/PhysRevA.103.062427

\section{INTRODUCTION}

Quantum operations with continuous variables represent a promising path towards scalable quantum computing and communication [1-4]. Like in qubit-based systems, a major challenge for faithful bosonic quantum information is to store, manipulate, and communicate information in the presence of noise, such as excitation loss, quadrature drift, dephasing, and cavity nonlinearities. To overcome excitation loss that fundamentally limits cavity lifetime, multimode codes were introduced [5-10]. Later, to utilize the large Hilbert space of a bosonic mode and perform hardwareefficient operations, single-mode codes for excitation loss such as cat codes [11-15], binomial (bin) codes [16], and the more generic rotation-symmetric bosonic codes [17] were developed. Meanwhile, progress in superconducting circuit quantum electrodynamics (cQED), e.g., real-time adaptive control [13], fault-tolerant readout of excitation parity [18,19], and universal control of cavity [20-23], has opened up possibilities once thought unreachable, including implementing arbitrary quantum channels $[24,25]$. With the advances, errorcorrected cat and bin qubits and the associated universal gate sets have been demonstrated [13,26-29]. These capabilities together make possible higher-level tasks with bosonic

\footnotetext{
*Present address: Joint Center for Quantum Information and Computer Science, NIST, University of Maryland, College Park, Maryland 20742, USA.
}

systems, such as gate teleportation [30] and distributing errorcorrected entangled states [31].

The aforementioned codes for excitation loss are designed in the Fock basis, with code words spanned by well-separated subsets of basis states (i.e., parity structure) - the protection against error comes entirely from the separation. Despite the clear physics, this approach of code construction uses the Hilbert space inefficiently, as only some of the degrees of freedom available are considered. In this work, we explore the conjugate degree of freedom to basis-state occupationthe phase carried by each basis-state component in code words - and demonstrate its critical role for efficient bosonic quantum error correction (QEC). First, by examining how phase-imparting unitaries propagate through error processes, we show that tuning the phase degree of freedom in bosonic codes can affect the protection against Markovian errors that involve excitation exchange with the environment. To illustrate the idea, we modify the encoding procedures for bin and cat codes with a Kerr unitary that periodically alters the sign of probability amplitudes in code words and show that, by creating destructive interference error code words, the sign alteration (SA) effectively suppresses bit-flip-type decoherence. A two-level recovery that utilizes both the parity structure and destructive interference is employed to yield enhanced restoration at practical loss rates. For quantum dynamics involving both energy exchange and mode nonlinearities, prior arts on encoding are rare. We employ biconvex optimization of encoding and decoding [32-34] to generate optimal codes from scratch and show the critical role of phase in achieving optimality. 


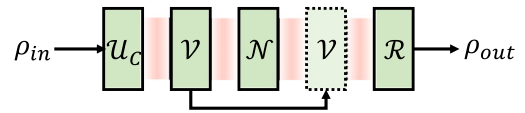

FIG. 1. Propagation of unitary channel $\mathcal{V}$ through error process $\mathcal{N}$. Successful propagation, as shown here, means it can be absorbed into $\mathcal{R}$ and has no effect on how an encoding protects against $\mathcal{N}$. Otherwise, $\mathcal{V}$ is a degree of freedom that can alter, and potentially enhance, the correction of $\mathcal{N}$.

Extending the principle beyond the Fock basis, we modify Gottesman-Kitaev-Preskill (gkp) codes defined in the position-momentum basis that protect against quadrature drift. We rediscover that gkp over nonrectangular lattices, which offers better protection than rectangular-lattice gkp, can be converted from the latter by SA [3]. For multiqubit codes, we phase engineer the nine-qubit Shor's code and achieve improved correction of Pauli errors and qubit amplitude damping. These examples demonstrate the generality of exploiting the phase degree of freedom to design efficient QEC schemes across physical platforms.

\section{PRINCIPLE}

A QEC process $\mathcal{E}$ consists of encoding $\mathcal{U}_{\mathrm{c}}$, noise $\mathcal{N}$, and recovery $\mathcal{R}$ and decoding $\mathcal{U}_{\mathrm{c}}^{\ddagger}$, i.e., $\mathcal{E}=\mathcal{U}_{\mathrm{c}}^{\ddagger} \circ \mathcal{R} \circ \mathcal{N} \circ$ $\mathcal{U}_{\mathrm{c}}$. As passive decoherence processes can mostly be treated as Markovian, we focus on Markovian dynamics with the Lindbladian generator $\mathcal{D}[\hat{A}](\hat{\rho})=\hat{A} \hat{\rho} \hat{A}^{\dagger}-\frac{1}{2}\left\{\hat{A}^{\dagger} \hat{A}, \hat{\rho}\right\} \quad(\hat{A}$ is the jump operator). If a unitary channel $\mathcal{V}(\rho)=\hat{V} \rho \hat{V}^{\dagger}$ can propagate through $\mathcal{N}=e^{\mathcal{D}[\hat{A}] t}$ (Fig. 1) and still end up unitary, i.e.,

$$
\mathcal{N} \circ \mathcal{V}=\mathcal{V} \circ \mathcal{N}
$$

it will not affect any QEC protocol on $\mathcal{U}_{\mathrm{c}}$, as $\hat{V}$ can be absorbed into $\mathcal{R}$. In Appendix A, we show that Eq. (1) holds if $\left[\hat{A}, \hat{H}_{V}\right]=r \hat{A}$, where $\hat{H}_{V}$ is the generating Hamiltonian for $\hat{V}$ and $r \in \mathbb{R}$. This criterion associates the error channel $\mathcal{N}$ with the set of unitaries that leave it invariant and helps identify quantum operations $\hat{V}$ that are transparent to error process $\mathcal{N}$. $\left[\hat{A}, \hat{H}_{V}\right]=0$ is the simplest way for $\hat{V}$ to be error transparent $[19,35-37]$, while, more generally, $\left[\hat{A}, \hat{H}_{V}\right]=r \hat{A}$ allows for path-independent quantum gates $[22,23]$.

On the other hand,

$$
\left[\hat{A}, \hat{H}_{V}\right] \neq r \hat{A}
$$

defines the set of $\hat{V}$ that alter how $\mathcal{U}_{\mathrm{c}}$ protects against $\mathcal{N}$. When separate evolution of $\hat{H}_{V}$ and $\hat{A}$ is achievable, Eq. (2) open the possibility of using $\mathcal{V}$ to enhance encoding $\mathcal{U}_{\mathrm{c}}$ and produce more distinguishable error states for $\mathcal{R}$ to recover. Specifically, for Markovian errors involving excitation exchange, excitation loss or gain, and thermal loss [34,38], we show that Eq. (2) is satisfied with phase-imparting Hamiltonians $\hat{H}_{V_{l}}=h_{l} \hat{n}^{l}(l \geqslant 2)$, where $\hat{n}=\hat{a}^{\dagger} \hat{a}$ is the bosonic number operator and $\hat{a}$ is the annihilation operator. Proper choices of $\hat{H}_{V_{l}}$ and interaction time can increase the separation between code words after errors and enable more faithful restoration (see Appendix A). We note that $\hat{V}$ should not be a logical gate on the original encoded subspace defined by $\mathcal{U}_{\mathrm{c}}$, as the code words (and hence QEC performance) will remain unaltered.
TABLE I. Maximum code-word overlap induced by $\hat{E}_{k}^{\dagger} \hat{E}_{S+k}$ for $\operatorname{bin}(5,5)$ and $\operatorname{sab}(5,5)$ at $\gamma=0.1$. The three leading terms $(k=0,1,2)$ are shown for comparison.

\begin{tabular}{lcc}
\hline \hline$\epsilon_{k}$ & $\operatorname{bin}(5,5)$ & $\operatorname{sab}(5,5)$ \\
\hline$k=0$ & $5.1 \times 10^{-2}$ & $2.5 \times 10^{-2}$ \\
$k=1$ & $2.8 \times 10^{-2}$ & $5.9 \times 10^{-3}$ \\
$k=2$ & $3.4 \times 10^{-3}$ & $1.8 \times 10^{-3}$ \\
\hline \hline
\end{tabular}

\section{SIGN-ALTERED bin CODE FOR EXCITATION LOSS}

Here, we apply the criterion [Eq. (2)] to protect against the excitation-loss channel $\mathcal{N}_{\gamma}$ ( $\gamma$ is the loss rate) generated by $\hat{a}$ and show that evolution under the Kerr Hamiltonian $\hat{H}_{\mathrm{Kr}}=$ $-K \hat{n}^{2} / 2$ allows the bin code to better overcome $\mathcal{N}_{\gamma}$.

In its operator-sum representation, $\mathcal{N}_{\gamma}(\rho)=\sum_{k} \hat{E}_{k} \rho \hat{E}_{k}^{\dagger}$, with the Kraus operator $\hat{E}_{k}=\sqrt{\frac{\gamma^{k}}{k !}}(1-\gamma)^{\frac{\hat{n}}{2}} \hat{a}^{k}$ associated with losing $k$ excitations $[5,15,16]$. Parametrized with $N$ and $S$, the original bin $(N, S)$ code encodes a qubit and corrects $S-1$ losses for $N \geqslant S$,

$$
\left|0_{\mathrm{bin}} / 1_{\mathrm{bin}}\right\rangle=\frac{1}{\sqrt{2^{N-1}}} \sum_{\text {peven/odd }}^{[0, N]} \sqrt{\left(\begin{array}{l}
N \\
p
\end{array}\right)}|p S\rangle .
$$

Applying $\hat{H}_{\mathrm{Kr}}$ to the encoded subspace for duration $t_{S}=$ $\pi / 2 K S^{2}$ implements the unitary $\hat{V}_{S}=e^{i \pi \hat{n}^{2} /(2 S)^{2}}$, which periodically flips the signs in $\left|0_{\mathrm{bin}}\right\rangle$ while keeping those in $\left|1_{\mathrm{bin}}\right\rangle$ unchanged (up to a global phase). As a result, we obtain the sign-altered binomial (sab) code with

$$
\left|0_{\text {sab }}\right\rangle=\frac{1}{\sqrt{2^{N-1}}} \sum_{\text {peven }}^{[0, N]}(-1)^{\frac{p}{2}} \sqrt{\left(\begin{array}{c}
N \\
p
\end{array}\right)}|p S\rangle
$$

and $\left|1_{\mathrm{sab}}\right\rangle=e^{i \frac{\pi}{4}}\left|1_{\mathrm{bin}}\right\rangle$.

With the same parity structure, the $\operatorname{sab}(N, S)$ code also corrects $S-1$ losses perfectly. The improvement emerges as we consider the overlap between higher-order error code words $\left\langle\mu_{\sigma}^{k} \mid \mu_{\bar{\sigma}}^{S+k}\right\rangle$, where $\left|\mu_{\sigma}^{k}\right\rangle:=\hat{E}_{k}\left|\mu_{\sigma}\right\rangle / \sqrt{\left\langle\mu_{\sigma}\left|\hat{E}_{k}^{\dagger} \hat{E}_{k}\right| \mu_{\sigma}\right\rangle}$. The parity structure guarantees $\left\langle\mu_{\sigma}^{k} \mid \mu_{\bar{\sigma}}^{k+l}\right\rangle=0$, with $l<S$, so $\left\langle\mu_{\sigma}^{k} \mid \mu_{\bar{\sigma}}^{S+k}\right\rangle$ are the leading terms contributing to a logical bit flip. In Table I, we consider $\operatorname{bin}(5,5)$ and $\operatorname{sab}(5,5)$ at $\gamma=0.1$ as an example and compare maximum error-word overlap,

$$
\epsilon_{k}:=\max \left\{\left|\left\langle\mu_{0}^{k} \mid \mu_{1}^{S+k}\right\rangle\right|,\left|\left\langle\mu_{1}^{k} \mid \mu_{0}^{S+k}\right\rangle\right|\right\},
$$

which upper bounds the amount of resulting $\hat{\sigma}_{x}$ and $\hat{\sigma}_{y}$ errors combined (i.e., logical bit-flip error). With direct substitution, one can see $(-1)^{\frac{p}{2}}$ in Eq. (4) induces destructive interference between error code words and reduces $\epsilon_{k}$, which typically is the leading type of decoherence for the amplitude-damping channel with equally spaced codes. We note that a faithful restoration also depends on correction of phase-flip error and will be examined by evaluating the widely adopted channel fidelity in the next section.

Experimentally, the Kerr unitary $\hat{V}_{S}$ can be applied either by storing the bin-encoded state in a Kerr cavity or using SNAP gates [20]. We note similar preprocessing-Gaussian 
presqueezing - was shown to improve the fidelity of nonGaussian states over lossy transmission $[39,40]$.

\section{TWO-LEVEL RECOVERY}

Faithful restoration of quantum information relies on a recovery adapted to the encoding and error process. With destructive interference between sab error code words, we need to incorporate an $\mathcal{R}$ that makes use of their enlarged separation. We first recall the original recovery proposed for bosonic codes with spacing $S[15,16,18]$ : The recovery $\mathcal{R}^{(1)}=$ $\left\{\hat{R}_{0}^{(1)}, \hat{R}_{1}^{(1)}, \ldots, \hat{R}_{S-1}^{(1)}\right\}$ has Kraus operator $\hat{R}_{i}^{(1)}=\hat{U}_{i}^{(1)} \hat{\Pi}_{i \bmod S}$, where $\hat{\Pi}_{i \bmod S}$ is the projection operator into the subspace with excitation number $i$ modulo $S$ and unitary $\hat{U}_{i}^{(1)}$ performs state transfer $\left|\mu_{\sigma}^{(S-i) \bmod S}\right\rangle \leftrightarrow\left|\mu_{\sigma}\right\rangle \cdot \mathcal{R}^{(1)}$ uses this parity structure to correct the first $S-1$ excitation losses, while losses beyond $S-1$ are incorrectly restored. As such, we call it "one-level" recovery.

For sab, we use a recovery $\mathcal{R}^{(2)}$ developed in Ref. [41] that, in addition to correcting the first $S-1$ losses, exploits the component in $\left|\mu_{\bar{\sigma}}^{S+k}\right\rangle(k=0,1, \ldots, S-1)$ orthogonal to $\left|\mu_{\sigma}^{k}\right\rangle:\left|\mu_{\bar{\sigma}}^{S+k}\right\rangle-\left\langle\mu_{\sigma}^{k} \mid \mu_{\bar{\sigma}}^{S+k}\right\rangle\left|\mu_{\sigma}^{k}\right\rangle$. Detailedly, the first level of $\mathcal{R}^{(2)}$, similar to $\mathcal{R}^{(1)}$ [16], uses the parity structure to fully correct the first $S-1$ losses. Each Kraus operator consists of a projection and a restoring unitary,

$$
\hat{R}_{k}^{(2)}=\sum_{\sigma}\left(\left|\mu_{\sigma}\right\rangle\left\langle\mu_{\sigma}^{k}\right|+\hat{U}_{k}^{\mathrm{res}}\right) \hat{P}_{k}
$$

Here, $k=0,1, \ldots, S-1, \hat{P}_{k}=\sum_{\sigma}\left|\mu_{\sigma}^{k}\right\rangle\left\langle\mu_{\sigma}^{k}\right|$ projects to each error subspace, and $\hat{U}_{k}^{\text {res }}$ finishes the unitary rotation in $\operatorname{Span}\left\{\left|\mu_{\sigma}\right\rangle,\left|\mu_{\sigma}^{k}\right\rangle\right\}$; for $k \neq 0$, it is simply $\left|\mu_{\sigma}^{k}\right\rangle\left\langle\mu_{\sigma}\right|$.

Also, $\mathcal{R}^{(2)}$ has a second level with $S$ Kraus operators,

$$
\hat{R}_{S+k}^{(2)}=\sum_{\sigma}\left(\left|\mu_{\bar{\sigma}}\right\rangle\left\langle v_{\bar{\sigma}}^{k}\right|+\hat{U}_{S+k}^{\mathrm{res}}\right) \hat{P}_{S+k},
$$

where, for $k=0,1, \ldots, S-1$, the normalized $\left|\nu_{\bar{\sigma}}^{k}\right\rangle \propto$ $\left|\mu_{\bar{\sigma}}^{S+k}\right\rangle-\left\langle\mu_{\sigma}^{k} \mid \mu_{\bar{\sigma}}^{S+k}\right\rangle\left|\mu_{\sigma}^{k}\right\rangle$ is recoverable and $\hat{P}_{S+k}=$ $\sum_{\sigma}\left|v_{\bar{\sigma}}^{k}\right\rangle\left\langle v_{\bar{\sigma}}^{k}\right|$. To make $\mathcal{R}^{(2)}$ a completely positive, tracepreserving (CPTP) map, we add $\hat{R}_{2 S+1}^{(2)}=\hat{M}^{\text {res }}\left(\hat{I}_{\mathcal{H}}-\sum_{i=0}^{2 S} \hat{P}_{i}\right)$, where $\hat{I}_{\mathcal{H}}$ is the identity operator on the bosonic Hilbert space and $\hat{M}^{\text {res }}$ is an arbitrary unitary acting on the complementary subspace of $\left\{\left|\mu_{\sigma}\right\rangle\right\} \cup\left\{\left|\mu_{\sigma}^{k}\right\rangle\right\} \cup\left\{\left|v_{\bar{\sigma}}^{k}\right\rangle\right\}$, where $\sigma=0,1$ and $0 \leqslant k \leqslant S-1$.

$\mathcal{R}^{(2)}$ was initially proposed for bin against excitation loss [41], yet the enhancement is much more pronounced for sab due to the enlarged separation between error words caused by destructive interference. Since $S$ to $2 S-1$ losses are now partially corrected, we call $\mathcal{R}^{(2)}$ "two-level" recovery. With a two-level ancilla coupled to the bosonic mode, recipes for constructing an arbitrary bosonic quantum channel have been proposed [24,25]. With rapid advances in superconducting cQED such as fault-tolerant readout [19] and cavity universal control $[20,21], \mathcal{R}^{(2)}$ is within reach of current technologies.

To quantify the overall error suppression of QEC protocols (i.e., both bit flip and phase flip), we adopt channel fidelitya specific entanglement fidelity-defined as $F:=\langle\Psi| \mathcal{I}_{A} \otimes$ $\mathcal{E}_{B}(|\Psi\rangle\langle\Psi|)|\Psi\rangle$ [41-43], where $\mathcal{I}$ is an identity channel and $|\Psi\rangle=(|00\rangle+|11\rangle) / \sqrt{2}$ is a Bell state. In Fig. 2, we present
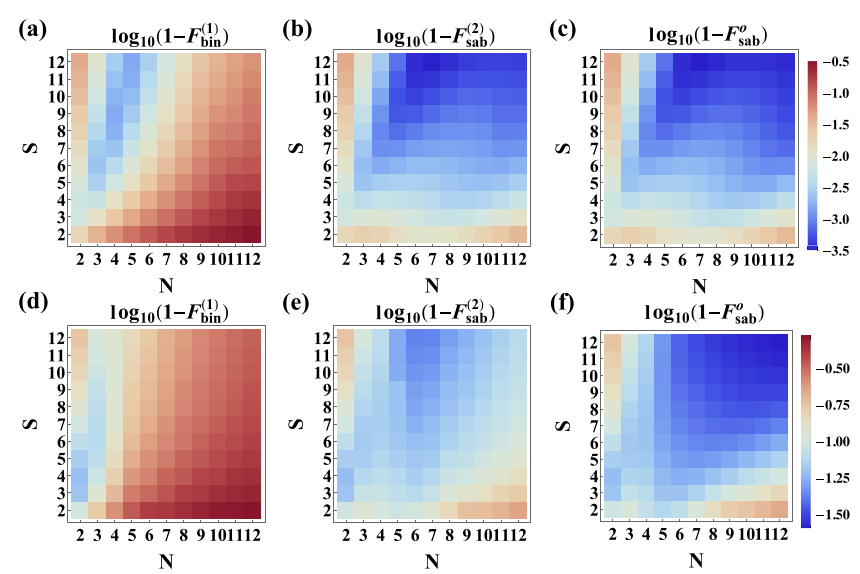

FIG. 2. Channel infidelities (in logarithmic scale) for (a)-(c) bin with $\mathcal{R}^{(1)}$, sab with $\mathcal{R}^{(2)}$, and sab with $\mathcal{R}^{\circ}$, respectively, at $\gamma=0.1$ and (d)-(f) same as (a)-(c), except $\gamma=0.25$. Each pixel is a code with associated $S$ and $N$.

the favorable performance of $\mathcal{R}^{(2)}$ and sab by comparing the channel infidelities $1-F$ for bin undergoing $\mathcal{N}_{\gamma}$ followed by $\mathcal{R}^{(1)}$ and those for sab undergoing the same $\mathcal{N}_{\gamma}$ followed by $\mathcal{R}^{(2)}$ and optimal recovery $\mathcal{R}^{0}$ obtained via convex optimization $[41,42]$, respectively. The optimal recovery $\mathcal{R}^{\circ}$ is numerically computed to maximize $F$ given a $\mathcal{U}_{c}$ and hence reveals the best-case performance of $\mathcal{U}_{c}$, yet it may lack physics intuition and ease of implementation, as it is restricted to be only CPTP [42].

We see from Fig. 2(a) that, at $\gamma=0.1$, the desired bin codes under $\mathcal{R}^{(1)}$ are found along $S \approx 2 N$, while the entire $N>S$ region is forbidden. In comparison, sab codes with $\mathcal{R}^{(2)}$ [Fig. 2(b)] achieve much lower infidelities overall and open up the $N>S$ region, clearly demonstrating the advantage of phase engineering for the creation of destructive interference and adapted recovery. Figure 2(c) shows the minimized channel infidelities for sab under $\mathcal{R}^{\circ}$. Comparing Figs. 2(b) and 2(c), we see that $\mathcal{R}^{(2)}$ works very well with sab at small $\gamma$, yielding near-optimal protection; the results hence demystify $\mathcal{R}^{\circ}$ for equally spaced codes: It is critical to resolve partially overlapped error words for faithful restoration. At a higher loss rate, $\gamma=0.25$ [Figs. 2(d)-2(f)], since $\mathcal{R}^{(2)}$ provides only two levels of correction, it begins to perform suboptimally in regions with excessive loss, i.e., $\bar{n} \gamma \gtrsim S$ [Figs. 2(f) and 2(e)], indicating the need to resolve higher-order error words for optimal restoration. Nonetheless, sab with $\mathcal{R}^{(2)}$ still significantly outperforms bin with $\mathcal{R}^{(1)}$, offering a robust and practically feasible bosonic QEC scheme against excitation loss.

cat is another equally spaced code and can be similarly enhanced, as detailed in Appendix B. Notably, the two-component cat code [12], the simplest of the code family with no correction of loss, will approximately correct one loss after SA. The same as sab, the SA can be implemented by $\hat{V}_{S}$, while now $2 S$ is the number of coherent states in superposition. $\hat{V}_{S}$ is not a logical gate on the cat code as it satisfies Eq. (2), and this explains why a proper amount of Kerr improves the cat code's performance ([41], Fig. 9(a)). 
TABLE II. Results from biconvex optimization under energy constraint $\bar{n}_{\mathrm{c}}=2$ for single-mode RCQCs and CCQCs at $\gamma=0.1$ and different values of intrinsic Kerr nonlinearity $K t$. Wigner functions of $\frac{1}{2} \hat{P}_{\mathrm{c}}$ are shown for the optimized codes, respectively, with associated channel infidelities $1-F$ below them. The infidelities for bin $(2,2)$ are shown as a benchmark.

\begin{tabular}{|c|c|c|c|c|}
\hline$K t$ & 0 & 0.5 & 1 & 1.5 \\
\hline RCQCs & 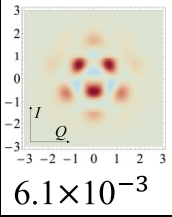 & 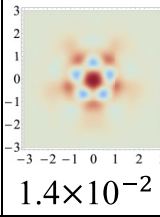 & 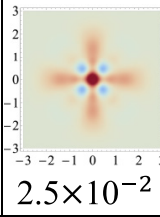 & 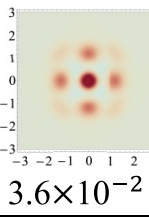 \\
\hline $\mathrm{CCQCs}$ & $\begin{array}{l}3 \\
2 \\
1 \\
0 \\
-2 \\
-3 \\
-3\end{array}$ & 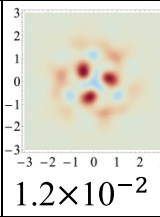 & 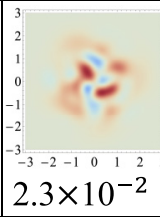 & $\begin{array}{l}\begin{array}{l}3 \\
2 \\
1 \\
0 \\
-1 \\
-2 \\
-3\end{array} \\
3.2 \times 10^{-2-1} \\
3.2 \times 10^{-2}\end{array}$ \\
\hline $\operatorname{bin}(2,2)$ & $1.8 \times 10^{-2}$ & $2.1 \times 10^{-2}$ & $3.0 \times 10^{-2}$ & $4.5 \times 10^{-2}$ \\
\hline
\end{tabular}

\section{OPTIMALITY VERSUS COMPLEX NOISE}

So far we have considered enhanced protection against excitation loss with sign-altered bosonic codes. In reality, when the bosonic mode is not perfectly harmonic, quantum dynamics are dictated jointly by energy-exchanging decoherence processes and cavity nonlinearities. As jumps resulted from decoherence can destroy the unitarity of evolution under cavity nonlinearities, these joint channels generally have complex, nonanalytical Kraus operators and hence are rarely studied despite their practical relevance. Here, we study such a channel-the joint channel of excitation loss and Kerrand show the critical role of the phase degree of freedom in optimal code construction.

The joint channel has the superoperator [44]

$$
\mathcal{N}_{\gamma, K t}=e^{-\frac{1}{2} i K t\left[\hat{n}^{2}, \cdot\right]-\ln (1-\gamma) \mathcal{D}[\hat{a}](\cdot)} .
$$

We use the technique of biconvex optimization of recovery and encoding for channel fidelity [32,33] to generate optimized encoding schemes. Specifically, we employ a variant that imposes an energy constraint on the encoding subspace to make the result practically feasible [34] (details in Appendix C). To see unambiguously the role of phase for optimal code construction, we compare the results for the optimized real-coefficient quantum code (RCQC) and complex-coefficient quantum code (CCQC), for which the code projector $\hat{P}_{\mathrm{c}}$ that fully characterizes a code is allowed to take arbitrary complex values.

In Table II we show the Wigner functions for the maximally mixed state $\frac{1}{2} \hat{P}_{\mathrm{c}}$ and associated infidelities $1-F$ of the optimized RCQCs and CCQCs at $\gamma=0.1$ and different $K t$ with an energy constraint $\bar{n}=2$. As a benchmark we show the infidelities for $\operatorname{bin}(2,2)\left(\bar{n}_{\mathrm{c}}=2\right)$, which protects against pure excitation loss [41]. For all $K t$, both scenarios yield codes better than bin $(2,2)$, demonstrating the advantage of channel adaptation achieved by biconvex optimization. With pure excitation loss (i.e., at $K t=0$ ) the optimized RCQC and
CCQC are equivalent, up to a global rotation. For $K t \neq 0$, nontrivial phases in CCQC allow for codes more efficient than RCQC. The results here demonstrate the benefit of mobilizing the entirety of degrees of freedom available to efficiently overcome practical noises.

\section{SIGN-ALTERED gkp AND MULTIQUBIT CODES}

While we have focused on codes defined in the Fock basis, the same idea of phase engineering applies to code constructs in other bases. For example, one can modify rectangular-lattice gkp code, defined in position-momentum space for quadrature drift [3], as $\left|\mu_{\sigma}\right\rangle=\sum_{s \in \mathbb{Z}}(-1)^{\sigma} \mid q=$ $\alpha(\sigma+2 s)\rangle$, where $\sigma=0,1$, and $2 \alpha$ is the spacing between position eigenstates. Similar to sab and sac, the SA (a symplectic transformation) can be imposed by following the original $\mathcal{U}_{\text {gkp }}$ with $\hat{V}=e^{i \pi \hat{q}^{2} / 4 \alpha^{2}}$ that transforms the original stabilizer $\hat{S}_{1}=e^{-i \hat{p} \alpha}$ to $\hat{S}_{1}^{\prime}=e^{-i\left(\hat{p}-\pi \hat{q} / 2 \alpha^{2}\right) \alpha}$ while leaving $\hat{S}_{2}=e^{2 \pi i \hat{q} / \alpha}$ unchanged. The new stabilizers define a nonrectangular gkp lattice, and at $\alpha^{2}=\sqrt{3} \pi / 2$ it is hexagonal: the optimal packing in two dimensions with a larger smallestuncorrectable shift $[3,34,45]$.

The interference effects further extend into the multiqubit regime. Consider Shor's [[9, 1, 3]] code that corrects arbitrary single-qubit Pauli errors with code words $\mid-$ shor $\rangle \propto|\tilde{1} \tilde{0} \tilde{0}\rangle+$ $|\tilde{0} \tilde{1} \tilde{0}\rangle+|\tilde{0} \tilde{0} \tilde{1}\rangle+|\tilde{1} \tilde{1} \tilde{1}\rangle$ and $\mid+$ shor $\left._{\text {shor }}\right\rangle=\hat{\sigma}_{x}^{\otimes 9}\left|-{ }_{\text {shor }}\right\rangle$, where $\tilde{i}=$ iii stands for blocks of three qubits. It detects weight-three $\hat{\sigma}_{x}$ errors, except for the logical operators $\hat{\sigma}_{x}^{(i)} \hat{\sigma}_{x}^{(i+1)} \hat{\sigma}_{x}^{(i+2)}$, with $i=1,4,7$. Consider a sign-altered variant with $\mid-$ shor' $\left.^{\prime}\right\rangle \propto$ $|\tilde{1} \tilde{0} \tilde{0}\rangle-|\tilde{0} \tilde{1} \tilde{0}\rangle+|\tilde{0} \tilde{0} \tilde{1}\rangle-|\tilde{1} \tilde{1} \tilde{1}\rangle$ and $\left|+_{\text {shor }^{\prime}}\right\rangle=\left|+_{\text {shor }}\right\rangle$. The new code, which is an example of code-word-stabilized code [46], detects all weight-three $\hat{\sigma}_{x}$ errors, including $\hat{\sigma}_{x}^{(i)} \hat{\sigma}_{x}^{(i+1)} \hat{\sigma}_{x}^{(i+2)}$, as well as more weight-three hybrid $\hat{\sigma}_{x}$ and $\hat{\sigma}_{y}$ errors, while also offering the same protection over $\hat{\sigma}_{z}$ as the original Shor code (more details are given in Appendix B). A similar modification improves Shor and Steane codes over qubit amplitude damping, a realistic concern for qubit systems [47]. We note that, for stabilizer codes, it has been shown to be adequate to consider RCQC $[48,49]$, so any nontrivial multiqubit CCQC will not be a stabilizer code.

\section{CONCLUSION}

In contrast to conventional designs of bosonic codes whose error-correction capability comes from separation between code words in the computational basis, we explored the conjugate degree of freedom, the phases carried by basis states, to devise efficient quantum codes for various errors. We showed it is possible to add phase-imparting unitaries to encoding procedures to enhance protection against Markovian errors involving excitation exchange. As illustrations, we considered the excitation-loss channel and modified binomial and cat codes with a Kerr unitary that makes the error code words destructively interfere and hence enlarges their separation. With a recovery that effectively captures the enlarged separation, the modified codes present noticeable improvement over the original ones. In the presence of mode nonlinearities, energy-exchanging decoherence processes can lead to complex quantum dynamics. We considered the joint channel 
of excitation loss and cavity Kerr and showed the critical role of phase for optimal code construction. Finally, we showed phase engineering extends beyond bosonic codes and can help multiqubit codes better overcome qubit errors.

With the general principle of phase engineering and explicit examples of qubit codes developed here, it should be straightforward to extend to codes that encode a qudit. Nonetheless, it remains to be systematically studied how the phase degree of freedom should be tuned for different encoding schemes and channels and how to construct associated recoveries that efficiently capture the enlarged distance between error words. We hope these developments will further deepen our understanding of channel-adapted quantum error correction and inspire more efficient code designs for various physical platforms.

\section{ACKNOWLEDGMENTS}

We thank L. Pryadko, P. Faist, M. Zhang, S. Zhou, and W. Ma for helpful discussions. We acknowledge support from the ARL-CDQI (Grants No. W911NF-15-2-0067 and No. W911NF-18-2-0237), ARO (Grants No. W911NF-18-10020 and No. W911NF-18-1-0212), ARO MURI (Grant No. W911NF-16-1-0349), AFOSR MURI (Grants No. FA955014-1-0052 and No. FA9550-15-1-0015), DOE (Grants No. DE-SC0019406), NSF (Grant No. EFMA-1640959), and the Packard Foundation (Grant No. 2013-39273). This work was done before K.N. joined AWS Center for Quantum Computing.

\section{APPENDIX A: PROPAGATION OF UNITARY CHANNEL THROUGH MARKOVIAN PROCESS}

We consider the conditions under which a unitary channel $\mathcal{V}(\rho)=\hat{V} \rho \hat{V}^{\dagger}$ can propagate through Markovian process $\mathcal{N}$, i.e.,

$$
\mathcal{N} \circ \mathcal{V}=\mathcal{V} \circ \mathcal{N}
$$

This is important as unitary channels that do not propagate through can be used to alter, and potentially enhance, how a given quantum code protects against $\mathcal{N}$. Here, we focus on the Markovian process generated by the Lindbladian generator $\mathcal{D}[\hat{A}](\hat{\rho})=\hat{A} \hat{\rho} \hat{A}^{\dagger}-\frac{1}{2}\left\{\hat{A}^{\dagger} \hat{A}, \hat{\rho}\right\}$; it models a wide array of error channels in bosonic systems. To find $\hat{V}$ that satisfy Eq. (A1), we consider the following equivalent condition:

$$
\mathcal{N} \mathcal{V} \mathcal{N}^{-1}=e^{\mathcal{D}[\hat{A}] t} e^{-i\left[\hat{H}_{V}, \cdot\right] \tau} e^{-\mathcal{D}[\hat{A}] t}=e^{-i\left[\hat{H}_{V}, \cdot\right] \tau},
$$

where $\hat{H}_{V}$ is the generating Hamiltonian for $\hat{V}$ and $\tau$ is the associated interaction time. $t$ is the duration of the Markovian channel $\mathcal{N}$; to not lose generality, Eq. (A2) should hold for any $t$. Using the Baker-Campbell-Hausdorff $(\mathrm{BCH})$ relation

$$
e^{C} e^{B} e^{-C}=e^{B+[C, B]+\cdots+\frac{1}{n !}[\underbrace{C,[C, \ldots[C, B] \ldots]]}_{n},}
$$

we can simplify Eq. (A2) and immediately identify the sufficient and necessary condition

$$
\left[\mathcal{D}[\hat{A}],\left[\hat{H}_{V}, \cdot\right]\right]=0 .
$$

The "sufficient" part can be seen via direct substitution, while the condition is also necessary as $\left.\left.\frac{1}{n !}[\underbrace{C,[C, \ldots[C}_{n}, B] \ldots\right]\right]$, with $n \geqslant 1$ in Eq. (A3) are higher orders in $t$ and the only possibility to have $\left.\left.\sum_{n=1}^{\infty} \frac{1}{n !}[\underbrace{C,[C, \ldots[C}_{n}, B] \ldots\right]\right]=0$ given any $t$ is $[C, B]=0$.

Expanding $\left[\mathcal{D}[\hat{A}],\left[\hat{H}_{V}, \cdot\right]\right](\rho)$ gives

$$
\begin{aligned}
{\left[\mathcal{D}[\hat{A}],\left[\hat{H}_{V}, \cdot\right]\right](\rho)=} & \mathcal{D}[\hat{A}]\left(\hat{H}_{V} \rho-\rho \hat{H}_{V}\right)-\left[\hat{H}_{V}, \mathcal{D}[\hat{A}](\rho)\right] \\
= & \hat{A}\left(\hat{H}_{V} \rho-\rho \hat{H}_{V}\right) \hat{A}^{\dagger} \\
& -\frac{1}{2}\left\{\hat{A}^{\dagger} \hat{A},\left(\hat{H}_{V} \rho-\rho \hat{H}_{V}\right)\right\} \\
& -\left[\hat{H}_{V}, \hat{A} \rho \hat{A}^{\dagger}-\frac{1}{2}\left\{\hat{A}^{\dagger} \hat{A}, \rho\right\}\right] \\
= & {\left[\hat{A}, \hat{H}_{V}\right] \rho \hat{A}^{\dagger}-\frac{1}{2}\left\{\hat{A}^{\dagger}\left[\hat{A}, \hat{H}_{V}\right], \rho\right\} } \\
& -\hat{A} \rho\left(\left[\hat{A}, \hat{H}_{V}\right]\right)^{\dagger}+\frac{1}{2}\left\{\left(\left[\hat{A}, \hat{H}_{V}\right]\right)^{\dagger} \hat{A}, \rho\right\} .
\end{aligned}
$$

As one can see, $\left[\mathcal{D}[\hat{A}],\left[\hat{H}_{V}, \cdot\right]\right](\rho)$ vanishes for any $\rho$ if

$$
\left[\hat{A}, \hat{H}_{V}\right]=r \hat{A} \quad r \in \mathbb{R} .
$$

This condition contains the special scenario $\left[\hat{A}, \hat{H}_{V}\right]=0$ (i.e., $r=0$ ), which is connected to the notion of error-transparent quantum operations [35,36] where quantum gates are designed such that they would not be disturbed by the occurrence of error.

In a bosonic system, $\hat{H}_{V_{l}}=h_{l} \hat{n}^{l}\left(l \in \mathbb{Z}^{+}\right)\left(\hat{n}=\hat{a}^{\dagger} \hat{a}\right.$ is the bosonic number operator, and $\hat{a}$ is the annihilation operator) imparts a phase to the logical subspace, and we show below that $\hat{H}_{V_{l}}$ can alter how quantum codes protect against bosonic channels that induce excitation exchange. Jump operators associated with excitation exchange can be generically expressed (in normal order) as $\hat{A}_{\downarrow}(p, q)=\hat{n}^{q} \hat{a}^{p}$ and $\hat{A}_{\uparrow}(p, q)=$ $\left(\hat{a}^{\dagger}\right)^{p} \hat{n}^{q}$. Consider $\hat{A}_{\downarrow}(p, q)$; then

$$
\begin{aligned}
{\left[\hat{n}^{q} \hat{a}^{p}, \hat{n}^{l}\right] } & =\hat{n}^{q}\left[\hat{a}^{p}, \hat{n}^{l}\right] \\
& =p \hat{n}^{q} \sum_{i=0}^{l-1}(\hat{n})^{l-1-i} \hat{a}^{p}(\hat{n})^{i} .
\end{aligned}
$$

One can see, except for $l=1$ (for which $\hat{H}_{V_{1}}$ generates a global rotation), $\left[\hat{A}_{\downarrow}(p, q), \hat{H}_{V_{l}}\right]$ will not be proportional to $\hat{n}^{q} \hat{a}^{p}$. Similarly, for $\hat{A}_{\uparrow}(p, q)$,

$$
\begin{aligned}
{\left[\left(\hat{a}^{\dagger}\right)^{p} \hat{n}^{q}, \hat{n}^{l}\right] } & =\left[\left(\hat{a}^{\dagger}\right)^{p}, \hat{n}^{l}\right] \hat{n}^{q} \\
& =-p \sum_{i=0}^{l-1}(\hat{n})^{l-1-i}\left(\hat{a}^{\dagger}\right)^{p}(\hat{n})^{i} \hat{n}^{q}
\end{aligned}
$$

will not be proportional to $\hat{n}^{q}\left(\hat{a}^{\dagger}\right)^{p}$ except for $l=1$. On the other hand, if $\hat{A} \propto \hat{n}^{l}$ (e.g., $A=\sqrt{\kappa_{\phi}} a^{\dagger} a$ for dephasing channel), the error channel does not involve excitation exchange with the environment and is naturally transparent to $\hat{H}_{V_{l}}$. In this case, phase engineering will not alter the performance of any code for the error channel.

These results indicate that it is possible to use $\hat{H}_{V_{l}}=$ $h_{l} \hat{n}^{l}(l>1)$ to engineer bosonic codes such that they can protect error channels that involve excitation exchange. In this work, we mainly focus on the excitation-loss channel with $\hat{A}_{\downarrow}(1,0)=\sqrt{\kappa} \hat{a}$ and the Kerr Hamiltonian $\hat{H}_{V_{2}}=-K \hat{n}^{2} / 2$.

We note that the most general case for a unitary channel $\mathcal{V}$ to keep its unitarity after propagation through $\mathcal{N}$ is $\mathcal{N} \circ \mathcal{V}=$ $\mathcal{U}(t) \circ \mathcal{N}$, where $\mathcal{U}(t)$ is a time-dependent, yet deterministic, 
unitary channel, which can be absorbed into the recovery. This would relax Eq. (A2) to $e^{\mathcal{D}[\hat{A}] t} e^{-i\left[\hat{H}_{V}, \cdot\right] \tau} e^{-\mathcal{D}[\hat{A}] t}=$ $e^{-i\left[\int_{0}^{\tau} \hat{H}_{U}\left(\tau^{\prime}\right) d \tau^{\prime}, \cdot\right]}$ for any $t$, which implicitly requires the resulting unitary channel to depend only on the duration of decoherence process and not on the specific timing of jump occurrences. This "path independence" has been studied in the context of error-transparent quantum operations [22]. There, however, error-transparent gates are conditioned on the initial and final states measured-different final states will result in different gates. With no measurement allowed here, we do not expect (but cannot assertively exclude) a criterion more general than Eq. (A5).

Finally, we consider generic Markovian dynamics with $M$ jump operators, for which the Liouville superoperator reads $\mathcal{L}(\rho)=\sum_{i=1}^{M} \mathcal{D}\left[\hat{A}_{i}\right](\rho)$. Following the same derivation, $\left[\mathcal{L}(\cdot),\left[\hat{H}_{V}, \cdot\right]\right](\rho)=\sum_{i=1}^{M}\left[\hat{A}_{i}, \hat{H}_{V}\right] \rho \hat{A}_{i}^{\dagger}-\frac{1}{2}\left\{\hat{A}_{i}^{\dagger}\left[\hat{A}_{i}, \hat{H}_{V}\right], \rho\right\}$ $-\hat{A}_{i} \rho\left(\left[\hat{A}_{i}, \hat{H}_{V}\right]\right)^{\dagger}+\frac{1}{2}\left\{\left(\left[\hat{A}_{i}, \hat{H}_{V}\right]\right)^{\dagger} \hat{A}_{i}, \rho\right\}$. One obvious solution for vanishing $\left[\mathcal{D}[\hat{A}],\left[\hat{H}_{V}, \cdot\right]\right](\rho)$ is an extension of Eq. (A5):

$$
\left[\hat{A}_{i}, \hat{H}_{V}\right]=r_{i} \hat{A}_{i} \quad r_{i} \in \mathbb{R}, i \in[1, M] .
$$

In addition, a vanishing $\left[\mathcal{D}[\hat{A}],\left[\hat{H}_{V}, \cdot\right]\right](\rho)$ is also possible if terms associated with different jump operators cancel out. For example, with $\mathcal{L}(\rho)=\mathcal{D}\left[\hat{A}_{1}\right](\rho)+\mathcal{D}\left[\hat{A}_{2}\right](\rho)$, $\left[\mathcal{D}[\hat{A}],\left[\hat{H}_{V}, \cdot\right]\right](\rho)$ vanishes if $\left[\hat{A}_{1}, \hat{H}_{V}\right]=\hat{A}_{2}$ and $\left[\hat{A}_{2}, \hat{H}_{V}\right]=$ $\hat{A}_{1}$. However, since each jump operator contains an error rate, only under unique combinations of error rates will terms associated with different jump operators cancel each other out.

\section{APPENDIX B: ADDITIONAL QUANTUM CODES FROM SIGN ALTERATION}

\section{Modified cat code for excitation loss}

Consider the simplest cat code $\left|\mu_{\sigma}^{\text {cat }}\right\rangle \propto \sum_{n=0}^{\infty}$ $\frac{\alpha^{2 n+\sigma}}{\sqrt{(2 n+\sigma) !}}|2 n+\sigma\rangle$ for $\sigma \in\{0,1\}$. This code does not detect even a single loss event $\hat{a}$, as $\hat{a}\left|\mu_{1}^{\text {cat }}\right\rangle \propto\left|\mu_{0}^{\text {cat }}\right\rangle$ and vice versa. However, a simple modification produces the sign-altered cat (sac) code words $\left|\mu_{\sigma}^{\mathrm{sac}}\right\rangle \propto \sum_{n=0}^{\infty} \frac{\alpha^{2 n+\sigma}}{\sqrt{(2 n+\sigma) !}}(-1)^{\sigma+1}|2 n+\sigma\rangle$ that do not significantly overlap upon a loss event. While $\hat{a}\left|\mu_{1}^{\text {sac }}\right\rangle$ is still in the support of $\left|\mu_{0}^{\text {sac }}\right\rangle$ (i.e., in the even Fock state subspace, the overlap between $\hat{a}\left|\mu_{1}^{\text {sac }}\right\rangle$ and $\left|\mu_{0}^{\text {sac }}\right\rangle$ is exponentially suppressed with $\alpha$ due to destructive interference. Thus, SA can extend a cat code that does not correct a loss error to one that approximately does. This behavior can also be understood in terms of bosonic coherent states $|\alpha\rangle$ (with $\hat{a}|\alpha\rangle=\alpha|\alpha\rangle$ ). In this basis, $\left|\mu_{0}^{\text {sac }}\right\rangle \propto|i \alpha\rangle+|-i \alpha\rangle, \quad\left|\mu_{1}^{\text {sac }}\right\rangle \propto|\alpha\rangle-|-\alpha\rangle$, and $\left\langle\mu_{0}^{\text {sac }}|\hat{a}| \mu_{1}^{\text {sac }}\right\rangle \rightarrow 0$ since the coherent states of $\left|\mu_{0}^{\text {sac }}\right\rangle$ are well separated from those of $\hat{a}\left|\mu_{1}^{\mathrm{sac}}\right\rangle$.

Generically, the code words of cat codes are cat states, superpositions of $2 S$ coherent states lying equidistantly on a circle in the phase space [15]. In the Fock basis, they can be expressed as

$$
\left|C_{\alpha}^{n}\right\rangle=\sqrt{\frac{2 S}{\mathcal{N}_{\alpha}^{n}}} \sum_{m=0}^{\infty} \frac{e^{-\frac{\alpha^{2}}{2}} \alpha^{n+2 m S}}{\sqrt{(n+2 m S) !}}|n+2 m S\rangle,
$$
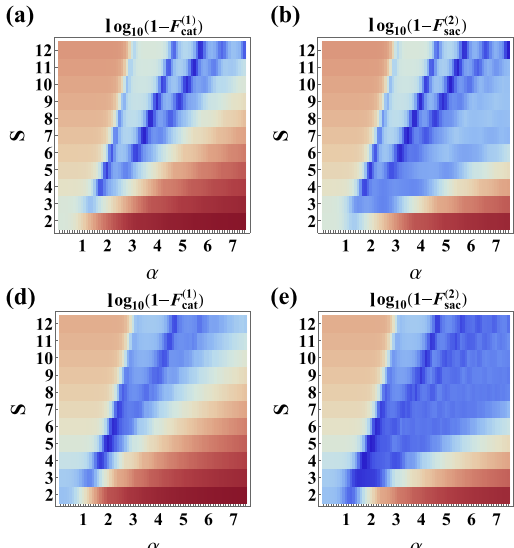

$\alpha$

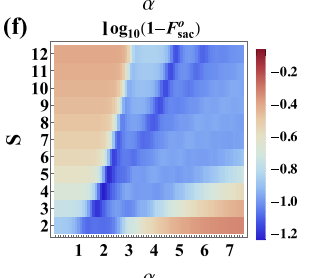

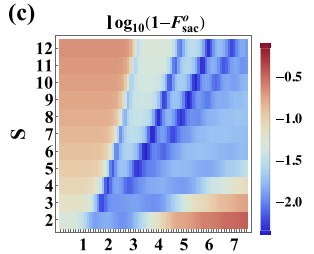

FIG. 3. Channel infidelities (in logarithmic scale) for (a)-(c) cat code with one-level recovery (as indicated by the superscript), sac code with two-level recovery, and sac code with optimal recovery, respectively, at $\gamma=0.1$ and (d)-(f) the same as (a)-(c), except for $\gamma=0.25$. Each point represents a code with associated $S$ and $\alpha$.

where $n=0,1, \ldots, 2 S-1, \mathcal{N}_{\alpha}^{n}=2 S\left\langle\alpha\left|\hat{\Pi}_{n \bmod s}\right| \alpha\right\rangle$ is the normalization factor, and $\lim _{\alpha \rightarrow \infty} \mathcal{N}_{\alpha}^{n}=1$. Without losing generality, $\alpha$ is assumed to be real here, and we choose $\operatorname{Span}\left\{\left|C_{\alpha}^{0}\right\rangle,\left|C_{\alpha}^{S}\right\rangle\right\}$ to be the logical subspace; for cat codes there is freedom in the choice of the " $d$ subspace," and $\operatorname{Span}\left\{\left|C_{\alpha}^{d}\right\rangle,\left|C_{\alpha}^{d+S}\right\rangle\right\}$ is the logical subspace ([15]).

The generic sac code is defined as follows:

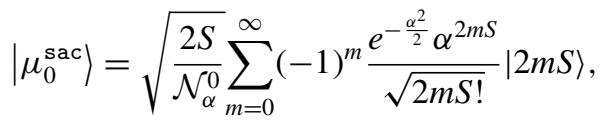

$$
\begin{aligned}
& \left|\mu_{1}^{\mathrm{sac}}\right\rangle=\sqrt{\frac{2 S}{\mathcal{N}_{\alpha}^{S}}} \sum_{m=0}^{\infty} \frac{e^{-\frac{\alpha^{2}}{2}} \alpha^{(2 m+1) S}}{\sqrt{(2 m+1) S !}}|(2 m+1) S\rangle .
\end{aligned}
$$

The above code words can be generated in the same way as the sab code, i.e., $\mathcal{U}_{\text {sac }}(\cdot)=\hat{V}_{\mathrm{s}} \mathcal{U}_{\text {cat }}(\cdot) \hat{V}_{\mathrm{S}}^{\dagger}$. We can see from Fig. 3 that, similar to sab, for sac the $\alpha^{2} \gamma \approx S$ region is opened up due to the additional suppression of errors induced by $S$ to $2 S-1$ losses and, at small loss, $\mathcal{R}^{(2)}$ yields a QEC performance close to optimal. Nonetheless, unlike bin codes, the performance of cat codes at finite $\alpha$ is constrained by dephasing caused by $\left\langle\mu_{0}^{\text {sac }}\left|\hat{n}^{m}\right| \mu_{0}^{\text {sac }}\right\rangle \neq\left\langle\mu_{1}^{\text {sac }}\left|\hat{n}^{m}\right| \mu_{1}^{\text {sac }}\right\rangle$ for $m \in \mathbb{Z}^{+}$. This is why at small loss rates the optimal codes are found around "sweet spots" (deep blue regions in Fig. 3), specific choices of $\alpha$ that minimize dephasing [15]. As a result, the SA-led suppression of bit-flip-type decoherence does not improve QEC performance as significantly as it does for sab codes.

\section{Modified Shor's $[[9,1,3]]$ code for Pauli errors}

Given a code with projection $P$ and error $E$, the error is said to be detectable if $P E P \propto P$. A set of errors $\mathbb{E}=\{E\}$ is said to correctable if $P E^{\dagger} F P \propto P$ for all $E, F \in \mathbb{E}$. Here, we showcase a sign-altered variant of the Shor code that better protects against Pauli errors. 
With stabilizers $\quad \sigma_{z}^{(1)} \sigma_{z}^{(2)}, \quad \sigma_{z}^{(2)} \sigma_{z}^{(3)}, \quad \sigma_{z}^{(4)} \sigma_{z}^{(5)}$, $\sigma_{z}^{(5)} \sigma_{z}^{(6)}, \quad \sigma_{z}^{(7)} \sigma_{z}^{(8)}, \quad \sigma_{z}^{(8)} \sigma_{z}^{(9)}, \quad \sigma_{x}^{(1)} \sigma_{x}^{(2)} \sigma_{x}^{(3)} \sigma_{x}^{(4)} \sigma_{x}^{(5)} \sigma_{x}^{(6)}$, and $\sigma_{x}^{(4)} \sigma_{x}^{(5)} \sigma_{x}^{(6)} \sigma_{x}^{(7)} \sigma_{x}^{(8)} \sigma_{x}^{(9)}$, the Shor code

$$
\begin{aligned}
\left|+_{\text {shor }}\right\rangle= & \frac{1}{2}(|000000000\rangle+|000111111\rangle \\
& +|111000111\rangle+|111111000\rangle), \\
\left|-{ }_{\text {shor }}\right\rangle= & \frac{1}{2}(|111000000\rangle+|000111000\rangle \\
& +|000000111\rangle+|111111111\rangle)
\end{aligned}
$$

corrects arbitrary single-qubit errors. For two-qubit errors, the code offers only partial correction as $\sigma_{x}^{(i)} \sigma_{x}^{(i+1)} \sigma_{x}^{(i+2)}$, where $i=1,4,7$ are logical $\sigma_{x}$ operators. We then consider the following modified Shor code:

$$
\begin{aligned}
\left|+_{\text {shor }^{\prime}}\right\rangle= & \frac{1}{2}(|000000000\rangle-|000111111\rangle \\
& +|111000111\rangle-|111111000\rangle), \\
\left|-{ }_{\text {shor }}\right\rangle= & \frac{1}{2}(|111000000\rangle+|000111000\rangle \\
& +|000000111\rangle+|111111111\rangle) .
\end{aligned}
$$

The stabilizer set of this code consists of the same six $\sigma_{z}$ stabilizers as the Shor code, $\sigma_{x}^{(1)} \sigma_{x}^{(2)} \sigma_{x}^{(3)} \sigma_{x}^{(7)} \sigma_{x}^{(8)} \sigma_{x}^{(9)}$ and $\sigma_{y}^{(1)} \sigma_{x}^{(2)} \sigma_{x}^{(3)} \sigma_{y}^{(4)} \sigma_{x}^{(5)} \sigma_{x}^{(6)} \sigma_{z}^{(7)}$. Its logical operators are $\bar{\sigma}_{x}=$ $\sigma_{z}^{\otimes 9}$ and $\bar{\sigma}_{z}=\sigma_{x}^{(1)} \sigma_{x}^{(2)} \sigma_{x}^{(3)} \sigma_{z}^{(4)}$, which is now weight-four. Like the Shor code, the modified code corrects arbitrary single-qubit errors and detects arbitrary two-qubit Pauli errors. In addition, one can see that

$$
\begin{aligned}
\sigma_{x}^{(1)} \sigma_{x}^{(2)} \sigma_{x}^{(3)}\left|+_{\text {shor' }}\right\rangle= & \frac{1}{2}(|111000000\rangle-|000111000\rangle \\
& +|000000111\rangle-|111111111\rangle), \\
\sigma_{x}^{(1)} \sigma_{x}^{(2)} \sigma_{x}^{(3)}\left|-_{\text {shor }}\right\rangle= & \frac{1}{2}(|000000000\rangle+|000111111\rangle \\
& +|111000111\rangle+|111111000\rangle),
\end{aligned}
$$

which leads to $P \sigma_{x}^{(1)} \sigma_{x}^{(2)} \sigma_{x}^{(3)} P=0$. In fact, the code now detects $E=\sigma_{x}^{(i)} \sigma_{x}^{(i+1)} \sigma_{x}^{(i+2)}$ for $i=1,4,7$ and hence all weight-three $\sigma_{x}$ errors. Similarly, one can check that fewer weight-three hybrid $\sigma_{x}$ and $\sigma_{y}$ errors are now undetectable. For error correction, one can also design recoveries to capture the additional QEC matrix elements that are now zero. The tradeoff for the improved error detection and correction is that the new code is no longer a Calderbank-Shor-Steane (CSS) code.

\section{Modified Shor's [[9, 1, 3]] code and Steane's [[7, 1, 3]] code for qubit amplitude damping}

For the single-qubit amplitude-damping channel with rate $\gamma$, the Kraus operators are

$$
\begin{gathered}
A_{0}=I+(\sqrt{1-\gamma}-1) \sigma_{+} \sigma_{-}, \\
A_{1}=\sqrt{\gamma} \sigma_{-},
\end{gathered}
$$

where $\sigma_{-}=|0\rangle\langle 1|=\sigma_{+}^{\dagger}$.

Consider the correction of qubit amplitude-damping errors with the Shor code in Eq. (B5). The logical code words can be conveniently expressed as $|-\rangle \propto|\tilde{1} \tilde{0} \tilde{0}\rangle+|\tilde{0} \tilde{1} \tilde{0}\rangle+$ $|\tilde{0} \tilde{0} \tilde{1}\rangle+|\tilde{1} \tilde{1} \tilde{1}\rangle$ and $\mid+$ shor $\rangle=\sigma_{x}^{\otimes 9}\left|-_{\text {shor }}\right\rangle$, where $\tilde{i}=i i i$ stands for blocks of three qubits. The code detects arbitrary twoqubit damping errors and ceases to protect against some of the three-qubit errors, such as $\tilde{\sigma}_{-}^{(i)}=\sigma_{-}^{(i)} \sigma_{-}^{(i+1)} \sigma_{-}^{(i+2)}$ for $i \in\{1,4,7\}$ and $\sigma_{-}^{(i)}=|0\rangle_{i}\left\langle\left. 1\right|_{i}\right.$. Now consider the modified code word $\mid-$ shor" $\left.^{\prime \prime}\right\rangle \propto|\tilde{1} \tilde{0} \tilde{0}\rangle+|\tilde{0} \tilde{1} \tilde{0}\rangle+|\tilde{0} \tilde{0} \tilde{1}\rangle-|\tilde{1} \tilde{1} \tilde{1}\rangle$. Taking $i=1$ as an example, $\tilde{\sigma}_{-}^{(1)}\left|{ }_{\text {shor" }}\right\rangle \propto|\tilde{0} \tilde{0} \tilde{1}\rangle-|\tilde{0} \tilde{1} \tilde{1}\rangle$ then does not overlap with $\left|+_{\text {shor }}\right\rangle=\left|+_{\text {shor }}\right\rangle$. While such errors cannot be fully detected as $\left\langle-_{\text {code }}\left|\tilde{\sigma}_{-}^{(i)}\right|+_{\text {code }}\right\rangle \neq 0$ for both versions of the code, it is possible to capture the enhancement with an analogous two-level quantum recovery developed for those single-mode codes.

Similarly, we can modify the logical code words of the Steane code by altering the coefficient of the |1111111) computational basis state from +1 to -1 :

$$
\begin{aligned}
\left|0_{\mathrm{stn}^{\prime}}\right\rangle= & \frac{1}{\sqrt{8}}(|0000000\rangle+|1010101\rangle+|0110011\rangle \\
& +|1100110\rangle+|0001111\rangle+|1011010\rangle \\
& +|0111100\rangle+|1101001\rangle), \\
\left|1_{\mathrm{stn}^{\prime}}\right\rangle= & \frac{1}{\sqrt{8}}(-|1111111\rangle+|0101010\rangle+|1001100\rangle \\
& +|0011001\rangle+|1110000\rangle \\
& +|0100101\rangle+|1000011\rangle+|0010110\rangle) .
\end{aligned}
$$

Just like the original Steane code, stn $^{\prime}$ can provably correct arbitrary single-qubit Pauli errors. Since a larger set of weight-three errors is now not completely detectable, stn' is a worse-performing code against local Pauli noise. However, it is slightly better performing when it comes to qubit amplitude damping.

Both the Steane code and stn' correct one amplitudedamping error and fail to correct two losses in the same way. A difference occurs when it comes to three-loss errors $\sigma_{-}^{(i)} \sigma_{-}^{(j)} \sigma_{-}^{(k)}$ : Such errors become detectable for stn', for example, $\sigma_{-}^{(2)} \sigma_{-}^{(4)} \sigma_{-}^{(6)}\left|1_{s t n^{\prime}}\right\rangle \propto-|1010101\rangle+|0000000\rangle$, which is orthogonal to $\left|0_{\operatorname{stn}^{\prime}}\right\rangle$. The same occurs for $\{i, j, k\}=$ $\{1,4,5\},\{3,4,7\}$ and five other combinations corresponding to the five remaining basis elements of $\left|1_{\operatorname{stn}^{\prime}}\right\rangle$. The remaining possible loss triples annihilate both $\left|0_{\text {stn }^{\prime}}\right\rangle$ and $\left|1_{\text {stn }^{\prime}}\right\rangle$. Therefore, all loss triples are detectable. However, the modification does not improve the code against two-loss errors, and we observe that the channel fidelity (calculated using optimal recovery) improves at most in the third decimal place

\section{APPENDIX C: CONVEX AND BICONVEX OPTIMIZATION OF CHANNEL FIDELITY}

\section{Channel fidelity and convex optimization for optimal recovery}

Given an encoding $\mathcal{U}_{\mathrm{c}}$ for an error process $\mathcal{N}$, one shall desire to find a recovery $\mathcal{R}$ that faithfully restores the corrupted quantum information; obviously, a good $\mathcal{R}$ will be highly adapted to $\mathcal{U}_{\mathrm{c}}$ and $\mathcal{N}$. While the definition of "faithfully" is not unique, a widely used metric that quantifies how channel $\mathcal{E}=\mathcal{U}_{\mathrm{c}}^{\ddagger} \circ \mathcal{R} \circ \mathcal{N} \circ \mathcal{U}_{\mathrm{c}}$ preserves quantum information is the channel fidelity $[32,41]$. Consider preparing two qubits, A and $\mathrm{B}$, in a maximally entangled state $|\Psi\rangle=\left(\left|0_{A} 0_{B}\right\rangle+\right.$ $\left.\left|1_{A} 1_{B}\right\rangle\right) / \sqrt{2}$ and then sending qubit $B$ through the qubit channel $\mathcal{E}$ (qubit A remains untouched). Channel fidelity of $\mathcal{E}$ is 
defined as

$$
F:=\left\langle\Psi\left|\mathcal{I}_{A} \otimes \mathcal{E}_{B}(|\Psi\rangle\langle\Psi|)\right| \Psi\right\rangle
$$

where $\mathcal{I}_{A}$ is an identity channel on qubit A. In its Kraus representation, $\mathcal{E}(\rho)=\sum_{i=1}^{4} A_{k} \rho A_{k}^{\dagger}$, and channel fidelity takes the form

$$
F=\frac{1}{4} \sum_{i=1}^{4}\left|\operatorname{Tr}\left[A_{k}\right]\right|^{2} .
$$

Compared to other fidelity measures that can be used to quantify how a channel preserves quantum information such as minimum fidelity [42,50] and code-word overlap [51], $F$ stands out as the computation of optimal recovery $\mathcal{R}^{\circ}$ that maximizes channel fidelity for $\mathcal{E}=\mathcal{U}_{\mathrm{c}}^{\ddagger} \circ \mathcal{R} \circ \mathcal{N} \circ \mathcal{U}_{\mathrm{c}}$ is a semidefinite programming problem and can be very efficiently solved [33,41-43]. Specifically, with the input map $\mathcal{N} \circ \mathcal{U}_{\mathrm{c}}$, which maps from the code subspace to the bosonic space, computation of the optimal $\mathcal{U}_{\mathrm{c}}^{\ddagger} \circ \mathcal{R}$-a reverse map-is a convex optimization. The fact that optimal channel fidelity can be easily computed in this way makes comparison of different encoding schemes for a given error channel easily trackable.
Regardless of the choice of channel measure, the numerically computed optimal recovery $\mathcal{R}^{\circ}$ only needs to be CPTP and hence in general can lack physical intuition and ease of implementation [42] - this is certainly the case for $\mathcal{R}^{\circ}$ obtained from convex optimization of channel fidelity. For more details of the convex optimization technique, we refer readers to Refs. [33,41-43].

\section{Biconvex optimization of channel fidelity for optimal encoding and recovery}

While computation of the optimal $\mathcal{U}_{\mathrm{c}}^{\ddagger} \circ \mathcal{R}$ that maximizes channel fidelity with $\mathcal{N} \circ \mathcal{U}_{\mathrm{c}}$ is a convex problem, given a recovery $\mathcal{R}$, computation of optimal encoding $\mathcal{U}_{\mathrm{c}}$ for $\mathcal{N}$ is also convex. With iterative optimization of recovery $\mathcal{R}$ and encoding $\mathcal{U}_{\mathrm{c}}$, after enough sampling for good convergence, one can identify the optimal encoding $\mathcal{U}_{\mathrm{c}}^{\mathrm{o}}$ and recovery $\mathcal{R}^{\circ}$ for $\mathcal{N}$ [33]. Recently, it was shown in Ref. [34] that, in the reverse optimization of $\mathcal{U}_{\mathrm{c}}$ given $\mathcal{R}$ and $\mathcal{N}$, one can apply an energy constraint to the encoding subspace while keeping the convexity of the problem. This allows us to obtain optimal encoding schemes under energy constraints that are practical in terms of energy requirements. We refer readers to Refs. [33,34] for more details.
[1] E. Knill, R. Laflamme, and G. J. Milburn, Nature (London) 409, 46 (2001).

[2] S. L. Braunstein and P. van Loock, Rev. Mod. Phys. 77, 513 (2005).

[3] D. Gottesman, A. Kitaev, and J. Preskill, Phys. Rev. A 64, 012310 (2001).

[4] T. C. Ralph, A. Gilchrist, G. J. Milburn, W. J. Munro, and S. Glancy, Phys. Rev. A 68, 042319 (2003).

[5] I. L. Chuang, D. W. Leung, and Y. Yamamoto, Phys. Rev. A 56, 1114 (1997).

[6] D. W. Leung, M. A. Nielsen, I. L. Chuang, and Y. Yamamoto, Phys. Rev. A 56, 2567 (1997).

[7] Y. Ouyang, Phys. Rev. A 90, 062317 (2014).

[8] M. Bergmann and P. van Loock, Phys. Rev. A 94, 012311 (2016).

[9] M. Y. Niu, I. L. Chuang, and J. H. Shapiro, Phys. Rev. A 97, 032323 (2018).

[10] Y. Ouyang and E. Campbell, arXiv:2008.12576.

[11] Z. Leghtas, G. Kirchmair, B. Vlastakis, R. J. Schoelkopf, M. H. Devoret, and M. Mirrahimi, Phys. Rev. Lett. 111, 120501 (2013).

[12] M. Mirrahimi, Z. Leghtas, V. V. Albert, S. Touzard, R. J. Schoelkopf, L. Jiang, and M. H. Devoret, New J. Phys 16, 045014 (2014).

[13] N. Ofek, A. Petrenko, R. Heeres, P. Reinhold, Z. Leghtas, B. Vlastakis, Y. Liu, L. Frunzio, S. M. Girvin, L. Jiang, M. Mirrahimi, M. H. Devoret, and R. J. Schoelkopf, Nature (London) 536, 441 (2016).

[14] M. Bergmann and P. van Loock, Phys. Rev. A 94, 042332 (2016).

[15] L. Li, C.-L. Zou, V. V. Albert, S. Muralidharan, S. M. Girvin, and L. Jiang, Phys. Rev. Lett. 119, 030502 (2017).
[16] M. H. Michael, M. Silveri, R. T. Brierley, V. V. Albert, J. Salmilehto, L. Jiang, and S. M. Girvin, Phys. Rev. X 6, 031006 (2016).

[17] A. L. Grimsmo, J. Combes, and B. Q. Baragiola, Phys. Rev. X 10, 011058 (2020).

[18] L. Sun, A. Petrenko, Z. Leghtas, B. Vlastakis, G. Kirchmair, K. M. Sliwa, A. Narla, M. Hatridge, S. Shankar, J. Blumoff, L. Frunzio, M. Mirrahimi, M. H. Devoret, and R. J. Schoelkopf, Nature (London) 511, 444 (2014).

[19] S. Rosenblum, P. Reinhold, M. Mirrahimi, L. Jiang, L. Frunzio, and R. J. Schoelkopf, Science 361, 266 (2018).

[20] S. Krastanov, V. V. Albert, C. Shen, C.-L. Zou, R. W. Heeres, B. Vlastakis, R. J. Schoelkopf, and L. Jiang, Phys. Rev. A 92, 040303(R) (2015).

[21] R. W. Heeres, B. Vlastakis, E. Holland, S. Krastanov, V. V. Albert, L. Frunzio, L. Jiang, and R. J. Schoelkopf, Phys. Rev. Lett. 115, 137002 (2015).

[22] W.-L. Ma, M. Zhang, Y. Wong, K. Noh, S. Rosenblum, P. Reinhold, R. J. Schoelkopf, and L. Jiang, Phys. Rev. Lett. 125, 110503 (2020).

[23] P. Reinhold, S. Rosenblum, W.-L. Ma, L. Frunzio, L. Jiang, and R. J. Schoelkopf, Nat. Phys. 16, 822 (2020).

[24] S. Lloyd and L. Viola, Phys. Rev. A 65, 010101(R) (2001).

[25] C. Shen, K. Noh, V. V. Albert, S. Krastanov, M. H. Devoret, R. J. Schoelkopf, S. M. Girvin, and L. Jiang, Phys. Rev. B 95, 134501 (2017).

[26] R. W. Heeres, P. Reinhold, N. Ofek, L. Frunzio, L. Jiang, M. H. Devoret, and R. J. Schoelkopf, Nat. Commun. 8, 94 (2017).

[27] L. Hu, Y. Ma, W. Cai, X. Mu, Y. Xu, W. Wang, Y. Wu, H. Wang, Y. P. Song, C. L. Zou, S. M. Girvin, L.-M. Duan, and L. Sun, Nat. Phys. 15, 503 (2019). 
[28] A. Joshi, K. Noh, and Y. Y. Gao, Quantum Sci. Technol. 6, 033001 (2021).

[29] W. Cai, Y. Ma, W. Wang, C. L. Zou, and L. Sun, Fundamental Research 1, 50 (2021).

[30] K. S. Chou, J. Z. Blumoff, C. S. Wang, P. C. Reinhold, C. J. Axline, Y. Y. Gao, L. Frunzio, M. H. Devoret, L. Jiang, and R. J. Schoelkopf, Nature (London) 561, 368 (2018).

[31] C. J. Axline, L. D. Burkhart, W. Pfaff, M. Zhang, K. Chou, P. Campagne-Ibarcq, P. Reinhold, L. Frunzio, S. M. Girvin, L. Jiang, M. H. Devoret, and R. J. Schoelkopf, Nat. Phys. 14, 705 (2018).

[32] M. Reimpell and R. F. Werner, Phys. Rev. Lett. 94, 080501 (2005).

[33] R. L. Kosut and D. A. Lidar, Quantum Inf. Process. 8, 443 (2009).

[34] K. Noh, V. V. Albert, and L. Jiang, IEEE Trans. Inf. Theory 65 , 2563 (2019).

[35] O. Vy, X. Wang, and K. Jacobs, New J. Phys. 15, 053002 (2013).

[36] E. Kapit, Phys. Rev. Lett. 120, 050503 (2018).

[37] Y. Ma, Y. Xu, X. Mu, W. Cai, L. Hu, W. Wang, X. Pan, H. Wang, Y. P. Song, C. L. Zou, and L. Sun, Nat. Phys. 16, 827 (2020).

[38] J. Eisert and M. M. Wolf, arXiv:quant-ph/0505151.
[39] R. Filip, Phys. Rev. A 87, 042308 (2013).

[40] H. Le Jeannic, A. Cavaillès, K. Huang, R. Filip, and J. Laurat, Phys. Rev. Lett. 120, 073603 (2018).

[41] V. V. Albert, K. Noh, K. Duivenvoorden, D. J. Young, R. T. Brierley, P. Reinhold, C. Vuillot, L. Li, C. Shen, S. M. Girvin, B. M. Terhal, and L. Jiang, Phys. Rev. A 97, 032346 (2018)

[42] A. S. Fletcher, arXiv:0706.3400.

[43] A. S. Fletcher, P. W. Shor, and M. Z. Win, Phys. Rev. A 75, 012338 (2007).

[44] Another format of Kerr Hamiltonian contains a linear part in the number operator. As phase rotation commutes with excitation loss (see Appendix A for details), the choice of format will not affect optimal code constructs..

[45] J. Harrington and J. Preskill, Phys. Rev. A 64, 062301 (2001).

[46] A. Cross, G. Smith, J. A. Smolin, and B. Zeng, IEEE Trans. Inf. Theory 55, 433 (2009).

[47] A. S. Darmawan and D. Poulin, Phys. Rev. Lett. 119, 040502 (2017).

[48] E. M. Rains, IEEE Trans. Inf. Theory 45, 2361 (1999).

[49] D. Gottesman, arXiv:quant-ph/9705052.

[50] E. Knill and R. Laflamme, Phys. Rev. A 55, 900 (1997).

[51] R. Wickert and P. van Loock, arXiv:1303.0279. 